\title{
Research on human subjects: Australian ethics committees take tentative steps
}

\author{
L W Osborne Austin Hospital, Victoria, Australia
}

\section{Author's abstract}

Australian medical researchers are attempting to formulate a response to some of the ethical issues in medical research. The debate over the in vitro fertilisation programme has highlighted some community concern about research ethics and the role of the ethics committee. While very little is known about Australian ethics committees, it appears that a two-tiered approach comprising both ethical review and scientific review is acceptable to the research community. However, this approach plus some problems with the nature of informed consent, begs the question of the role of these committees in the broader context of medical research in the community. Important aspects of a seminar for members of hospital ethics committees are reported.

Only recently has the protection of human subjects of medical research been discussed extensively in Australia. Although Australia has an active medical research programme, there seemed no grounds for believing that there were any conflicts of interest between medical researchers and their subjects. More recently discussion of the ethical aspects of medical research has been illustrated by the community interest in the in vitro fertilisation programme, by press reports of the use of human pancreatic tissue as a laboratory testing system, by transplantation of tissues to children and adults, by the use of fetal bone to measure environmental radiation levels and by a community survey of the carcinogenic potential of a herbicide that required access to patients' records.

It was against this background that the Health Commission of Victoria held the first ever meeting for members of hospital ethics committees. From the papers presented at the seminar and from the discussion, it was clear that real efforts were being made to develop an approach to human subjects research that will be right for the Australian community.

Two-tiered review - the ethics committee and the medical research committee

Many different views were expressed on the role and Key words

Medical ethics; medical research; research ethics committees; consent; proxy consent; in vitro fertilisation. the structure of the ethics committees in Australian institutions. This diversity of opinion is not surprising since formal ethics committees were only established in most institutions in the late 1960s after the National Health and Medical Research Council (NHMRC) issued its own guidelines regarding the conduct of human experimentation. These guidelines outlined the ethical principles which were to be addressed by researchers when using human subjects in research projects and included such matters as disclosure of risks and benefits, consent procedures and the level of competence of the researchers. Of special importance was the statement that before any research could be funded by the NHMRC, the research protocol had to be approved by an institutional ethics committee and evidence of this approach forwarded to the NHMRC.

Within these very general guidelines there was plenty of scope for variation in approach. The most interesting variation discussed at the meeting was the relationship between the ethics committee and the medical research committee.

The predominant view was that the ethics committee should consider only the ethical or moral aspects of the protocol and should not consider the scientific merit of the proposed research. In order to fulfil this role the committee should be composed of non-medical people who would be able to bring community perspectives and values to any contentious moral issues raised by the research. These community representatives would be drawn from the members of the board of management of the hospital and from the ranks of the legal and religious professions. Medical and scientific experts would not be members of the committee but could be consulted if their expertise was required.

The medical research committee was seen as the place for the discussion of the technical and scientific aspects of the protocol. Since this committee would be composed entirely of medical researchers, the prospects of the project advancing knowledge could be assessed. If the project was not thought to be feasible given the institution's resources or was not consistent with the research priorities of the institution or had a poorly designed protocol, then the research committee would not approve it. The other major function of the research committee was to compile a register of projects being conducted in the institution. 
A case study of the working relationships between the two committees was given by one major hospital which had adopted the two-tier system. This ethics committee had prepared its own code of conduct of human subjects research and had issued a statement to researchers regarding the interpretation of the code. The hospital believed that with these guidelines the research committee could adequately review all projects for ethical propriety, referring to the ethics committee only those projects that appeared to be particularly sensitive. In addition, the ethics committee sampled the protocols approved by the research committee on an ad hoc basis in order to determine the extent of compliance with the ethics committee's guidelines.

Some discussants thought that the ethics committee acting as the conscience of the research committee in this way reduced the value of the committee in protecting the interests of all subjects. This faction argued that all protocols should be reviewed in detail if the interests of all subjects were to be protected. The additional administrative burdens and any possible delays in the implementation of research involved in reviewing all projects were considered to be the costs of knowing that the interests of all subjects had been considered prior to their being recruited for a project.

The meeting did not consider the main theoretical objection to the two-tiered arrangement which is that medical research is enshrined as a fundamental good that cannot be challenged by the non-scientific community. It seems that a community perspective about the need, value or worthwhileness of a particular research project cannot be considered in this dual system since only the medical researchers will consider the merit of the project. The ethical dilemmas associated with human subjects research will be presented by the researchers only in terms of the level of risk to which it is justifiable to submit subjects. Thus, the common concern expressed in America regarding the morality of putting subjects to any risk if the project does not add to the body of useful medical knowledge, has yet to surface in Australia. In fact, the two-tiered approach may retard considerations of this kind.

The Australian community is not yet prepared to debate the value of medical research as an end in itself. This reticence may relate to the success of a campaign aimed at both the community and politicians, which was conducted by medical researchers to increase the financial support for medical research.

It is clear that the merits, or otherwise, of the twotier system have not been fully explored. Only as further information becomes available about the practices in different hospitals can the feasibility of the two-tier system for the protection of Australian research subjects be evaluated. Other subtle aspects of the committee system on research will also have to be monitored.

\section{Consent forms - content and context}

The seminar expressed a real sense of frustration about informed consent procedures. While the legal opinion was expressed that the law did not wish to frustrate genuine scientific advances, the participants assessed the community's mood as being 'to sue first and ask questions later'. Many hospitals had responded to this litigious climate by insisting that consent forms were to be completed whenever subjects were recruited for research protocols.

The researchers felt that this approach was very superficial since it gave undue emphasis to the content of the form rather than to the context of the research. It was argued that the form could not take into account all the exigencies of the proposed research and especially the risks of participation. In many circumstances the risks of participation could not be known and hence could not be disclosed to the potential subjects. Even considering the known risks of some procedures, some patients' lack of English and their education or cultural backgrounds meant it would be extremely difficult for the researcher to be convinced the subject had understood all aspects of his or her involvement. This 'written consent is meaningless' group felt that the process was a sham and that they were being dishonest to themselves and to their institutions by their involvement in the process.

The response to this criticism was two-fold. Some wished to have a standard consent form for all protocols which would give the potential subject sufficient detail about the particular project as well as providing standard details, such as the ability to opt out of the protocol, the voluntary nature of the consent, and the extent of the explanation given about the project by the principal investigator. The form should also dictate that sufficient time be given to discuss the matter with family or friends. The more extreme position was that since no documentation was ever satisfactory the hospital had to rely upon the conscience of the investigator to ensure that no patients would be used in a study unless the investigator was satisfied that the subject understood what was proposed. In these circumstances, the ethics committee's role was to make sure that individual investigators were aware of their moral and legal responsibilities. The committee's role was not to impose a rigid set of protocols about informed consent procedures or to endorse particular consent forms.

It may be possible to develop a consensus between these two views. Some research projects that are not especially intrusive, for example, those requiring the collection of small amounts of blood or that do not put subjects at special risk should be able to be conducted without special consent forms. The American experience has shown that it is possible to define such a group of projects which do not require formal prospective review by the full ethics committee. Projects which are not specified in this group would require formal review by the ethics committee and the committee would give particular emphasis to the development of the par- 
ticular consent form. This approach means that the researcher retains a considerable degree of autonomy with respect to the recruitment of subjects while at the same time the competing interest of protecting the subjects of higher risk research can be reviewed by the ethics committee.

\section{Difficulties with minority groups}

Some of the problems of informed consent to involvement in experimentation were crystallised when the use of minority groups was considered.

The central body in Victoria responsible for mental health research reviews those projects that require the participation of both voluntary and involuntary mental health patients. Some of the difficulties have been highlighted by requests from university researchers and other groups to use mental health subjects in drug trials. It has been recognised that many of these patients' consent to involvement is invalidated because of the severity of their illness, for example, by patients stating that they did not care if they died as a result of the new drug, since they did not deserve to be alive.

Because of these and related difficulties, consent from the patient is sought by the medical staff caring for the patient and a judgment exercised regarding the validity of that consent. Currently, substituted consent from a relative is not acceptable for participation in a research project.

Substituted consent for the participation of children in research was considered to be essential. The role of the ethics committee was to ensure that the informed consent by parents or guardians had been obtained prior to recruiting the child for the research study. The sensitivity of the matter was acknowledged since it was suggested that a doctor not involved in the care of the child should seek the parents' consent in order to protect the parents from undue anxiety about their decision to allow their child to participate. It was recognised that the child should assent to his or her involvement whenever possible.

Finally, consent to in vitro fertilisation procedures was considered. The ethics committee of the Queen Victoria Medical Centre established an ad hoc committee to review many of the issues associated with in vitro fertilisation and embryo transfer. The guidelines issued by that committee emphasised that consent should be obtained from the potential parents at all stages of the fertilisation process and that the couple must have the option of withdrawal at any time. In addition it was recognised that parents should give consent to any procedure which was to be conducted on the zygote.

While the philosophical basis for some of these concerns was not well developed at the meeting, it was very clear that the participants were able to detect the tension between respecting the rights of the minority subjects, and not limiting the ability of researchers to conduct research that may be of benefit to these groups. It was argued that ethics committees should be involved in the development of a community consensus on the issues as well as in justifying decisions to the public. The previous assumption that any research that had been passed by an ethics committee would be consistent with general community values had to be reconsidered following the present debate in Australia over the in vitro fertilisation programme.

\section{Future developments}

It will be interesting to watch the development of the ethics committees in Australia. The little knowledge we have about the committees indicates that there are many different expectations of their role in the regulation of research. It may be that since the philosophical basis for the protection of research subjects has been poorly explored in Australia, the committees may not see their primary role as the protection of the human subjects of medical research. Since the committees were established as a requirement of the main research funding body, they may adopt a very pragmatic stance on the protection issue. In fact, this pragmatism may extend to the facilitation of research by committees keeping regulations to a minimum and through their promotion of research to the community.

The other implication of the committees' lacking philosophical sophistication is that the researchers may come to see the committees as making ethical decisions for them and absolving them of their moral or legal responsibilities.

The consent form is the main instrument that the committees see available to them in their protective brief. However, the consent form is recognised as a blunt instrument since the initiative remains more with the researcher than the subject. Apparently there are very few ways in which the consent form can be modified to alter the power imbalance between subject and researcher and attempts to make the form more detailed often result in a complex document expressed in pseudo-legal jargon which is meaningless to both subjects and researchers.

It would be unfortunate if Australian medical research was constrained in innovation and development through a misunderstanding of the law's interest in these matters. In Australia, some of the less satisfactory aspects of the American approach to litigation in routine medical practice are being promoted by the media and it will be very easy for these notions to be transferred to the research area.

A balance to this legalism could be struck if the ethics committees saw their function as alerting the researchers to the potential moral problems of research as well as educating researchers to the practical aspects of research ethics by providing a community review setting. Finally, the committees will have greater community credibility if they can encourage the researchers to look at the value of their research in terms of the nation's health needs. 